\title{
Titanium dioxide catalytic activity contributes to the process of free radical scavenging
}

\section{Maria Canillasa, , Eva Chinarroa, Marta Freitasb,c, Ana P. Pêgób,c,d,e, Berta Morenoa}

a Instituto de Cerámica y Vidrio, ICV-CSIC, C/Kelsen 5, 28049, Spain

b Instituto de Investigação e Inovação em Saúde, Universidade do Porto, Portugal

c INEB - Instituto de Engenharia Biomédica, Universidade do Porto, Porto, Portugal

d Faculdade de Engenharia da Universidade do Porto (FEUP), Porto, Portugal

e Instituto de Ciências Biomédicas Abel Salazar (ICBAS), Universidade do Porto, Portugal

*Corresponding author.E-mail address:mcanillas@icv.csic.es(M. Canillas).

Originally published in Journal of Catalysis Volume 381, January 2020, Pages 186-192 https://doi.org/10.1016/j.jcat.2019.09.030

\begin{abstract}
$\mathrm{TiO}_{2}$ crystallochemical properties have been explored and correlated with the activity of this material in the scavenging of reactive oxygen and nitrogen species (ROS/RNS). It is well known that those highly reactive species are produced by inflammatory cells and neutralized by some oxides, such a titanium dioxide under light. Nevertheless, the mechanism of action of this ceramic material is yet to be fully understood. A set of reactions have been proposed that imply the presence of different valence states of titanium as the basis for the ROS/RNS scavenging, but in this work, we have demonstrated that those species are not always detected in $\mathrm{TiO}_{2}$-based materials, which are, in addition, active in depleting ROS/RNS. $\mathrm{TiO}_{2}$ powders with undetected $\mathrm{Ti}_{3}+$ have been obtained with a range of different properties-surface area, particle size, rutile-anatase ratio, and band gap values-by varying the powder processing conditions after synthesis through the sol precipitation route. The properties of the powders have been correlated with the scavenging activity of the materials toward two reactive species, DPPH. and peroxynitrite, always conducted under dark conditions. It was observed that despite the lack of $\mathrm{Ti}_{3}+$ states, $\mathrm{TiO}_{2}$ powders can still neutralize ROS/RNS. For the first time, this study explain how the catalytic activity of different $\mathrm{TiO}_{2}$ contribute to the free radical scavenging mechanism and the differences in scavenger activity shown by different $\mathrm{TiO}_{2}$ phases and mixtures.
\end{abstract}




\section{Keywords}

Titanium dioxide; ROS/RNS; Peroxynitrite; DPPH ; Heterojunction

\section{INTRODUCTION}

Reactive oxygen and nitrogen species (ROS/RNS) are commonly involved in modulating effects on inflammation and play a key role in the regulation of the immune responses [1], [2]. Inflammation is required for the regeneration of tissues and healing, but in some cases, it has deleterious effects [3]. It also contributes significantly to several physiologic events, such as aging, which is associated with high levels of oxidized biomolecules [4], [5]. Despite the existence of an antioxidant defense system that counteracts oxidative damage by ROS/RNS, such modifications in proteins, lipids, and DNA have been proposed as the starting point in the development of age-dependent diseases. Among the described ROS/RNS, peroxynitrite is a powerful oxidizing agent that results from the reaction between superoxide and nitric oxide radicals. In fact, nitric oxide (NO) plays a regulatory role at every stage of inflammation. This molecule is generally oxidized within seconds to peroxynitrite, which mediates the cytotoxic effects of NO, causing damage similar to that observed with hydroxyl radicals [6], [7].

It was previously demonstrated that $\mathrm{TiO}_{2}$ is able to neutralize both ROS and RNS produced by inflammatory cells as mediators during the inflammatory response [8], [9]. Usually, for most prostheses and nondegradable implants, the inflammation process ends with the encapsulation of the material. But in the case of titanium-based implants, this inflammatory response is cushioned by $\mathrm{TiO}_{2}$ through the neutralization of ROS/RNS, which avoids their final encapsulation [10]. Suzuki has reported previously that passivated Ti surfaces decrease the reactivity of the inflammatory mediator peroxynitrite in vitro, even in the presence of bicarbonate and plasma proteins [8]. They suggested, in accordance with Tengvall, the importance of the creation of a Ti peroxygel on the surfaces of $\mathrm{Ti}$ implants [9]. Subsequently, the Frangos team suggested the important role of $\mathrm{TiO}_{2}$ in this mechanism and proposed a set of redox reactions that involved two different valence states for titanium in a regenerative mechanism that depletes both peroxynitrite and superdioxide [11]. The scavenging properties of $\mathrm{TiO}_{2}$-based materials have been associated with the presence of crystallographic defects in the $\mathrm{TiO}_{2}$ surfaces [12]. To prove this hypothesis, $\mathrm{TiO}_{2}$ and $\mathrm{Sr} / \mathrm{Ba}$ titanates, undoped and Nb-doped, were tested, and the scavenging activity has been found to be directly correlated with the concentration of $\mathrm{Ti}_{3}+$ species in the sample.

Despite these contributions, the complete mechanism of ROS/RNS scavenging mediated by titanium oxides remains unknown at the moment. The mechanism proposed so far is based on the occurrence of redox reactions that imply changes in the crystal structure of the material, which can only take place under hard redox conditions that do not exist in physiological environments [13]. Furthermore, it also suggests the obligatory existence of reduced valence states in titanium. While the greater presence of reduced valence has been associated with an important increase in scavenging activity, these states are not always present, but the scavenging process occurs, as the current study demonstrates. 
Moreover, it has not been determined if there is any relationship between the different crystallographic structures of $\mathrm{TiO}_{2}$ and the ROS/RNS depletion activity. Rutile is thermodynamically more stable and crystallographically denser than anatase. Low-temperature synthesis of nanocrystalline $\mathrm{TiO}_{2}$ generally results in anatase nanoparticles that are transformed to rutile on reaching a certain size [14], [15]. Surface coordination, which inherently determines the heterogeneous reactivity of the powders, is different depending on the morphology and the crystallographic phase. Therefore, rutile and anatase can be synthesized with morphologies that favor certain facets with higher percentages of undercoordinated atoms and thus different reactivity [16].

With the aim of contributing to the understanding of the ROS/RNS scavenging process and to the unravelling of the mechanism behind it, we have studied the scavenging activity of $\mathrm{TiO}_{2}$ powders with controlled phase content and specific surface area toward two different radical species, peroxynitrite and DPPH (1,1-diphenyl-2-picryl-hydrazy radical), a semistable radical used in the determining the antioxidant activity of different molecules [17].

This work also aims to understand the roles of the different titanium coordinative environments to the process of ROS/RNS scavenging. This information will contribute to the use of $\mathrm{TiO}_{2}$ in the design of biomaterials with tuned anti-inflammatory properties.

\section{Experimental}

\subsection{Synthesis of TiO2 powders}

$\mathrm{TiO}_{2}$ was synthesized by the precipitation route, as previously described [18]. Ti (IV) isopropoxide (Sigma-Aldrich, 95\%) was dissolved in absolute ethanol at $70^{\circ} \mathrm{C}$ for $24 \mathrm{~h}$ under constant stirring. Afterward, hydrolysis took place in the presence of humidity. These precipitated powders were dried at room temperature, sieved in a 100- $\mu \mathrm{m}$ mesh, and then calcined in air at 200, 400,600,650,700, and $750^{\circ} \mathrm{C}$ for $12 \mathrm{~h}$. Once calcined, the powders were processed as follows: Their particle size was reduced for 5 h using an attrition mill in 2-propanol (Sigma-Aldrich, 95\%) and then the powders were sieved again. This procedure ensures the homogeneity of the particle size distribution and the high purity of the powders.

The calcination temperatures were chosen according to the phase changes and particle size growth. At $600{ }^{\circ} \mathrm{C}$, anatase-to-rutile phase transformation starts, while at $800^{\circ} \mathrm{C}$, only rutile is found in the powders. For these reasons, two samples, $\mathrm{TiO}_{2}$ calcined at 200 and $400^{\circ} \mathrm{C}$, have been chosen to study the influence of the particle size of the anatase phase on the $\mathrm{TiO}_{2}$ scavenging activity. Powders calcined at $600,650,700$, and $750^{\circ} \mathrm{C}$ were chosen to study the effect of the anatase-rutile ratio on the radical depletion capacity.

\subsection{Characterization techniques}

Identification of the crystallographic phases and the structure of $\mathrm{TiO}_{2}$ nanoparticles (powders) was carried out using a D-5000 X-ray diffraction (XRD) diffractometer (Siemens) operated at $50 \mathrm{kV}$ and 
$30 \mathrm{~mA}$, with CuKa radiation and a Ni filter, within the $2 \theta$ range $10-70^{\circ}$. The scanning step was $0.05^{\circ}$, the time/step was $1.5 \mathrm{~S}$, and the rotation speed used was $15 \mathrm{rpm}$. The crystalline phase percentage was calculated from the XRD pattern following the method described by Zachariah [19]. Narrow-scan analysis was conducted within the $2 \theta$ range $20^{\circ}-30^{\circ}$, as the main peaks of anatase $\mathrm{TiO}_{2}$ and rutile $\mathrm{TiO}_{2}$ are found within this range. The calculated percentages are semiquantitative. Specific surface area was measured by the B.E.T. method in an MS-13 monosorb analyzer (Quantachrome). Particle size distribution was determined with a Mastersizer laser diffraction analyzer (Malvern). Transmission electron microscopy (TEM) and high-resolution TEM (HRTEM) observations were carried out in an Hitachi $\mathrm{H}-7100$ working at an accelerating voltage of $125 \mathrm{kV}$ and a JEM $3000 \mathrm{~F}$ microscope (JEOL) operating at $300 \mathrm{kV}$ with a point resolution of $1.7 \AA$.

The band gap of the $\mathrm{TiO}_{2}$ powders was determined from UV-vis spectra acquired using a Lambda 950 UV-Vis-IRC spectrophotometer (Perkin Elmer). The measurements were carried out in the diffuse reflectance (DR) mode, at wavelengths between 200 and $1000 \mathrm{~nm}$. The Kubelka-Munk transformation was used for further calculations. The band gap was determined using a Tauc plot where the absorption coefficient $(\alpha)$ was related to the photon energy (hv) [20], [21]. The powders calcined at $600{ }^{\circ} \mathrm{C}$ were characterized by X-ray photoelectron spectroscopy (XPS) with a VG Escalab 200R spectrometer.

\subsection{Scavenging capacity of the Ti02 powders}

The scavenging activity of the powders was evaluated using two different radicals-peroxynitrite and 1,1-diphenyl-2-picryl-hydrazy radical (DPPH)-in assays based on previously described protocols [12], [22], [23].

DPPH is a semistable radical, which absorbs in the wavelength range corresponding to purple color. In the presence of anti-oxidative agents, this radical is neutralized to $\mathrm{DPPH}_{2}$, with a color change in the solution from purple to yellow (reduced form). The DPPH concentration was measured at $517 \mathrm{~nm}$. Although DPPH is not a physiological radical, the relatively low reaction kinetics allows a straightforward evaluation of the powders' reactivity. The different $\mathrm{TiO}_{2}$ samples (50 $\mathrm{mg}$ ) were incubated in a $0.1 \mathrm{mM}$ DPPH solution (Sigma-Aldrich) in 2-propanol $(500 \mu \mathrm{l})$ under protection from light, to avoid the scavenging effect of $\mathrm{TiO}_{2}$ in the presence of light and to simulate physiological lighting conditions, in a thermoblock set at $21^{\circ} \mathrm{C}$ under stirring (1200 rpm) for $30 \mathrm{~min}$. Subsequently, the suspension was centrifuged at relative centrifugal force ( $r c f) 20,000$ to sediment the powders, and $200 \mu \mathrm{l}$ of the supernatant was removed and its absorbance at $517 \mathrm{~nm}$ determined (Power Wave $\mathrm{XS}, \mathrm{BIOTEC}$ ). Results are expressed as the percentage of DPPH eliminated in relation to the DPPH added (determined with a powder-free 0.1-mM DPPH solution in 2-propanol).

3-Morpholinosidnominine hydrochloride ( $\mathrm{SIN}-1$ hydrochloride) spontaneously degrades in aqueous solutions to produce the oxygen free radicals superoxide anion and nitric oxide, which react in the medium to form peroxynitrite (ONOO-). The different $\mathrm{TiO}_{2}$ samples ( $50 \mathrm{mg}$ ) were incubated in $0.5 \mathrm{ml}$ of $25-\mu \mathrm{M}$ SIN-1 hydrochloride (Sigma-Aldrich) dissolved in 1-mM phosphate-buffered saline (PBS) and, to mimic a more physiologic environment, incubated in an electrolyte solution with the composition $\mathrm{KCl} 5 \mathrm{mM}, \mathrm{NaCl} 120 \mathrm{mM}, \mathrm{CaCl}_{2} 1.4 \mathrm{mM}, \mathrm{MgCl}_{2} 1.2 \mathrm{mM}$, dextrose $5 \mathrm{mM}$, and PBS $0.2 \mathrm{mM}$ [24]. In both cases, the mixtures were protected from light to avoid the scavenging effect of $\mathrm{TiO}_{2}$ in the presence of light and to simulate physiological lighting conditions in a thermoblock set at 
$21^{\circ} \mathrm{C}$ under stirring (1200 rpm) for $30 \mathrm{~min}$. The concentration of free radicals in solution after the incubation period was determined by fluorimetry using 6-carboxy-2 ' $7^{\prime}$ ' dichlorodihydrofluorescein diacetate (Carboxy-H2DCFDA; Molecular Probes, Life Technologies) previously deacetylated in $\mathrm{KOH} 0.1 \mathrm{M}$ (1:4), which, in the presence of ROS/RNS, will convert into a green-fluorescent form, $2^{\prime}, 7^{\prime}$-dichlorofluorescein (DCF). After the incubation period, samples were centrifuged at $\mathrm{rcf} 20,000$, while kept at $21^{\circ} \mathrm{C}$, and subsequently $150 \mu$ of the supernatant was taken and mixed with $50 \mu$ of a carboxy-H2DCFDA solution prepared in $1 \mathrm{mM}$ of PBS and $0.1 \mathrm{M} \mathrm{KOH}$, and the fluorescence was determined in a SPECTRAmax (Gemini XS, Molecular Devices) $(\lambda e x c=485 \mathrm{~nm} ; \lambda e m i=528 \mathrm{~nm})$.

Results are expressed as the percentage of ONOO- eliminated in relation to the ONOO- generated (determined in a powder-free SIN-1 solution).

\subsection{Statistics}

Data were analyzed using one-way ANOVA analysis of variance (SPSS 14.0 for Windows, SPSS, Chicago, IL) and the statistical difference was considered to be significant at $p<0.05$.

\section{Results and discussion}

Table 1, Table 2 summarize the crystallochemical and physical characterizations of the prepared materials. All of them were labeled with a code that starts with an $\mathrm{S}$, which means "sample," followed by the calcination temperature (So to S750), and R, which means the percentage of rutile phase, followed by the corresponding percentage in the sample, as determined by XRD ( $\mathrm{S}_{750} \mathrm{R}$ - 98 ).

Table 1. Summary of the crystallo-chemical characterization of the calcined powders based on XRD measurements.

\begin{tabular}{llll}
\hline Sample & Code & Rutile [Wt.\%] & Anatase [Wt.\%] \\
\hline $\mathrm{TiO}_{2} 200{ }^{\circ} \mathrm{C}$ & S200-R0 & 0 & 100 \\
$\mathrm{TiO}_{2} 400{ }^{\circ} \mathrm{C}$ & $\mathrm{S} 400-\mathrm{R0}$ & 0 & 100 \\
$\mathrm{TiO}_{2} 600^{\circ} \mathrm{C}$ & $\mathrm{S} 600-\mathrm{R} 23$ & 23 & 77 \\
$\mathrm{TiO}_{2} 650^{\circ} \mathrm{C}$ & $\mathrm{S} 650-\mathrm{R} 87$ & 87 & 13 \\
$\mathrm{TiO}_{2} 700^{\circ} \mathrm{C}$ & $\mathrm{S} 700-\mathrm{R} 97$ & 97 & 3 \\
$\mathrm{TiO}_{2} 750^{\circ} \mathrm{C}$ & S750-R98 & 98 & 2 \\
\hline
\end{tabular}


Table 2. Summary of the physical characterization of the calcined powders.

\begin{tabular}{lllll} 
Sample & Code & Particle Size Distribution $[\mu \mathrm{m}]$ & SSA $\left[\mathrm{m}^{2} / \mathrm{g}\right]^{\mathrm{a}}$ & Band-Gap [eV] \\
\hline $\mathrm{TiO}_{2} 200^{\circ} \mathrm{C}$ & $\mathrm{S} 200-\mathrm{R} 0$ & $0.2-50(15)^{\mathrm{b}}$ & $111 \pm 5$ & 3.40 \\
$\mathrm{TiO}_{2} 400^{\circ} \mathrm{C}$ & $\mathrm{S} 400-\mathrm{R0}$ & $0.2-50(10)^{\mathrm{b}}$ & $54 \pm 3$ & $10 \pm 1$ \\
$\mathrm{TiO}_{2} 600^{\circ} \mathrm{C}$ & $\mathrm{S} 600-\mathrm{R} 23$ & $0.5-50(2,10)^{\mathrm{b}}$ & $1.9 \pm 0.1$ & 3.32 \\
$\mathrm{TiO}_{2} 650^{\circ} \mathrm{C}$ & $\mathrm{S} 650-\mathrm{R} 87$ & $0.5-80(2,20)^{\mathrm{b}}$ & $1.2 \pm 0.1$ & 3.10 \\
$\mathrm{TiO}_{2} 700^{\circ} \mathrm{C}$ & $\mathrm{S} 700-\mathrm{R} 97$ & $0.5-80(2,20)^{\mathrm{b}}$ & $1.4 \pm 0.1$ & 3.03 \\
$\mathrm{TiO}_{2} 750^{\circ} \mathrm{C}$ & $\mathrm{S} 750-\mathrm{R} 98$ & $0.5-100(2,50 \uparrow)^{\mathrm{C}}$ & &
\end{tabular}

a Specific Surface Area.

b Maximum in the Particle Size Distribution. One value indicates a monomodal distribution, two values point to a bimodal distribution.

c $\uparrow$ Indicates that in a bimodal distribution, most of the particles shows a particle size distribution with 50 microns average.

XRD spectra were used to identify and quantify the crystal phases. Fig. 1 shows the patterns found for the powders after the different calcinations. It is noticeable how the broad bands at low angle values that are found in pattern (a) enhance their crystallinity when increasing temperature. At $400^{\circ} \mathrm{C}$ (Fig. 1b) the reflections of the anatase phase are clearly identified in the spectra, while at $600^{\circ} \mathrm{C}$ (Fig. 1C) the rutile phase is found mixed at $23 \%$ wt. with anatase. The phase transition between anatase (low-temperature phase) and rutile (high-temperature phase) is known to be dependent on the synthesis method and the particle size [14], [15]. In this work, at temperatures above $600{ }^{\circ} \mathrm{C}$, the powders show rutile as the main crystal phase, with a minor contribution from anatase (Figs. $1 d, 1$, and 1f). The percentage of rutile was found to increase exponentially from the lowest calcination temperatures $\left(<650^{\circ} \mathrm{C}\right)$ on, reaching the highest value for the samples calcined at the highest temperature tested. 


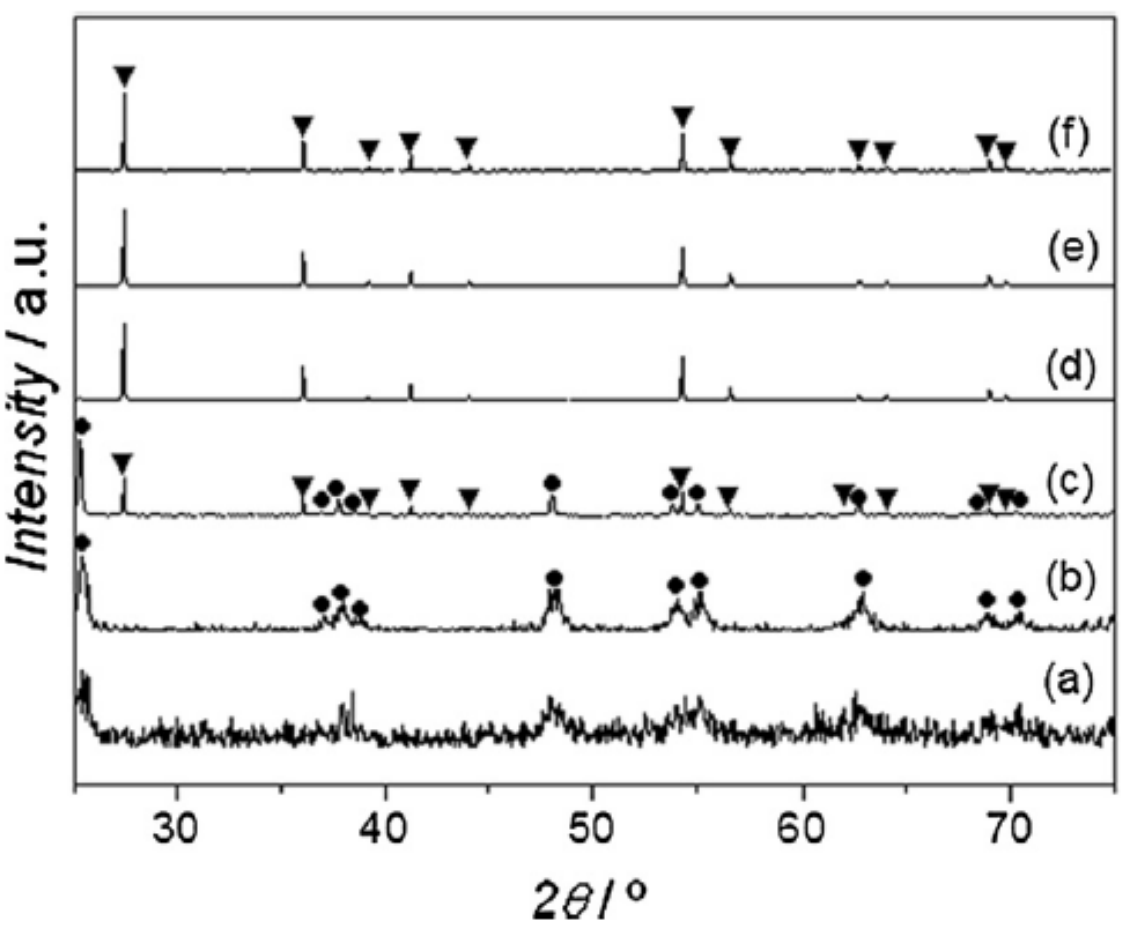

Fig. 1. XRD patterns of the TiO2 powders: (a) S200-Ro, (b) S400-Ro, (c) S60o-R23, (d) S650-R87, (e) S700-R97, and (f) S750-R98. Crystalline phases are identified: anatase $(\mathbf{)})$ and rutile ( $\mathbf{v}$ ). A.u.: arbitrary units.

From the particle size distributions measured and listed in Table 2, it can be observed that agglomerates are formed during the synthesis and calcination of the powders, as the values measured for particle size are between 0.2 and $100 \mu \mathrm{m}$, depending on the calcination temperature chosen. In fact, when the $\mathrm{TiO}_{2}$ powders undergo calcination (between 200 and $750^{\circ} \mathrm{C}$ ), grains grew in a bimodal particle size distribution with maxima between 1 and $10 \mu \mathrm{m}$, independent of the applied calcination temperature. However, TEM observations (Fig. 2a) in S200-Ro and S600-R23 show individual particle sizes of 17 and $34 \mathrm{~nm}$, respectively, and confirm the presence of agglomerates of $\mathrm{TiO}_{2}$ particles. 

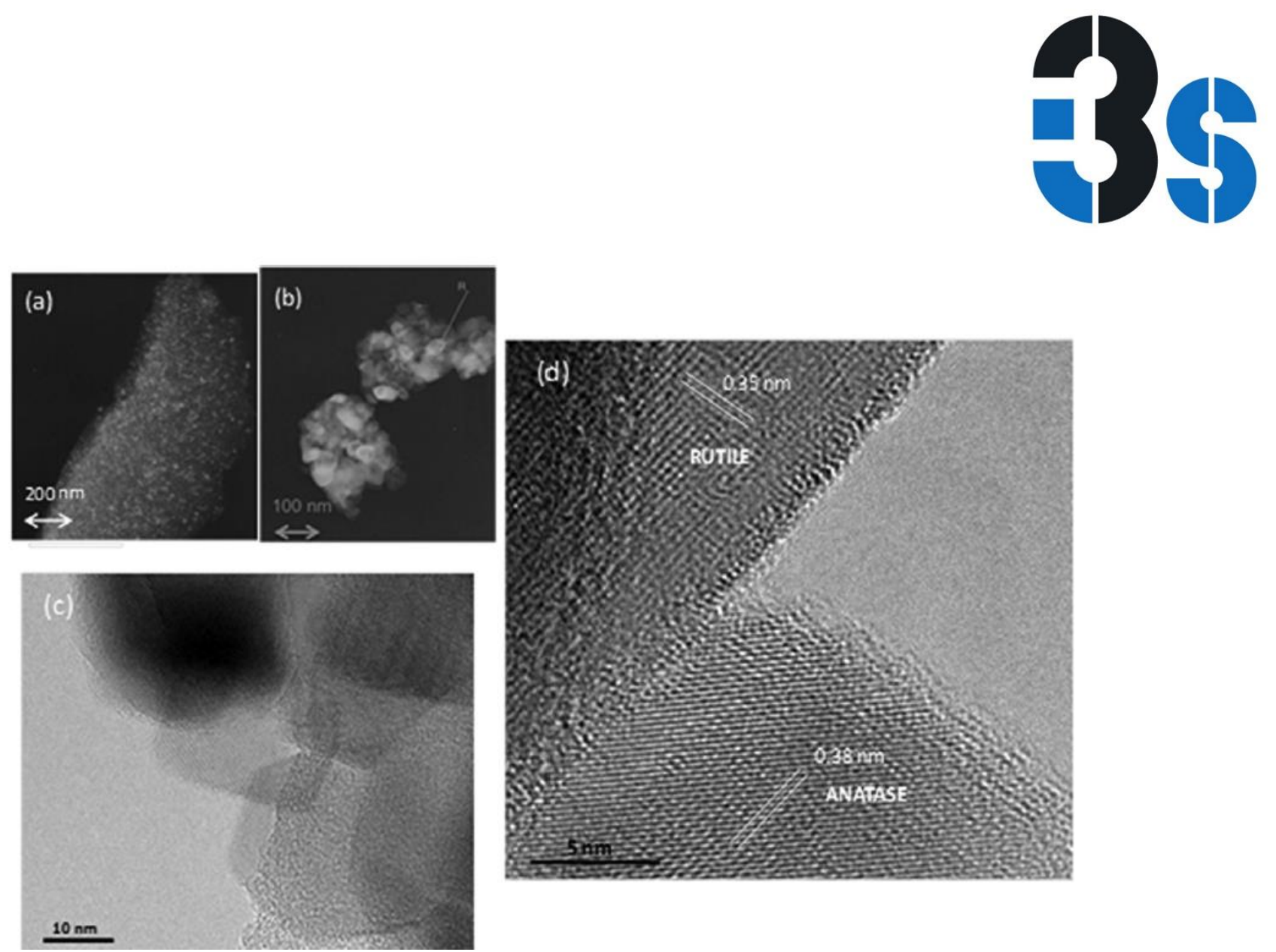

Fig. 2. TEM observations of (a) S200-Ro and (b) S60o-R23, powders show agglomeration of smaller particles. (c) HRTEM images of the $\mathrm{TiO}_{2}$ powders calcined at $600^{\circ} \mathrm{C}(\mathrm{S} 600-\mathrm{R} 23)$ and (d) detail of the anatase-rutile heterojunction with planar spaces assignment.

The specific surface areas (SSAs) of the powders were also found to be strongly affected by the calcination temperature and, as might be expected, by the particle size increase. In these samples, the values of SSA decreased progressively with temperature, reaching a stationary value for the treatment conducted at $650^{\circ} \mathrm{C}$. At $600^{\circ} \mathrm{C}$, particle size measured by TEM (Fig. 2b) is around $30 \mathrm{~nm}$. The increase of SSA is an important parameter that will be carefully taken into account during the results analysis.

The HRTEM images of the S600-R23 samples are shown in Figs. $2 b$ and $2 c$. The particle size was confirmed to be 20-30 nm, while two different morphologies for the particles can be distinguished. Particles with a less ordered structure and rounded shape have been ascribed to anatase, and others with a polygonal shape have been identified as rutile. Fig. $2 \mathrm{~d}$ shows an anatase and rutile heterojunction where the particle located above has been identified as a (1 1 10$)$ rutile facet, as the lattice fringes show a distance of $0.35 \mathrm{~nm}$. The particle located below has been associated with the (1 01 ) facet of anatase with a lattice distance of $0.38 \mathrm{~nm}$. These values are higher than reported for other rutile and anatase particles [25], [26], [27]. These discrepancies have been associated with the low crystallinity of the particles, which can increase the lattice distance as a consequence of structural disorder. The importance of such heterojunctions for the reactivity of the powders, among others in their photochemistry, has already been reported [28]. 
The band gap is the other parameter that was calculated from the UV-vis spectra of the samples, which are depicted in Fig. 3 . It is important to notice that the absorption edge changes in the different samples and that the band gap values (Table 2 ) decrease as the calcination temperature increases. The highest value was measured for the powders calcined at $200{ }^{\circ} \mathrm{C}(3.40 \mathrm{eV})$, in accordance with others reported in the literature and associated with the anatase phase $(3.20 \mathrm{eV})$ []. With calcination, the percentage of rutile is increased in the $\mathrm{TiO}_{2}$ samples and the band gap measured decreases proportionally until $3.00 \mathrm{eV}$, the value calculated for the $750^{\circ} \mathrm{C}$ sample, which is in agreement with the published data of $3.03 \mathrm{eV}$ [27]. All this information suggests that there is no $\mathrm{Ti} 3$ + presence varying the band gap value of the material.

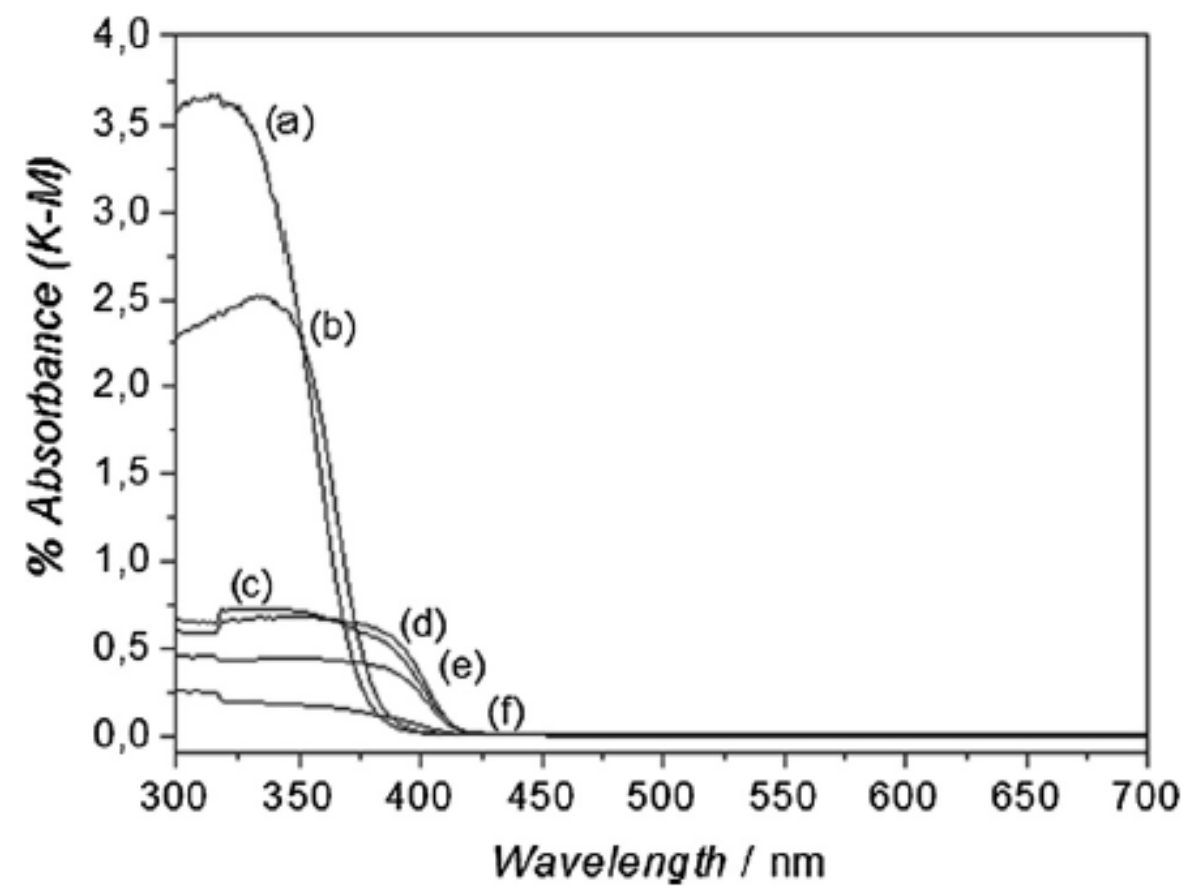

Fig. 3. UV-vis spectra (Kubelka-Munk absorbance vs. wavelength) of the prepared $\mathrm{TiO}_{2}$ samples: (a) S200-Ro, (b) S400-Ro, (c) S600-R23, (d) S650-87R, (e) S700-97R, and (f) S750-98R.

An XPS spectrum, representative of the Ti2p component in the surface of S6oo-R23, is depicted in Fig. 4. The binding energy value measured at $458.3 \mathrm{eV}$ corroborates that the spectrum is only composed by the contribution of $\mathrm{Ti}_{4}+$ in an oxide environment, discarding the presence of $\mathrm{Ti}_{3}+$, which should appear at lower binding energies (around $456 \mathrm{eV}$ ). This is also confirmed by the binding energy value of $529.9 \mathrm{eV}$ measured in the spectrum of oxygen (not shown), which depicts only one contribution, associated with the presence of $\mathrm{TiO}_{2}$. 


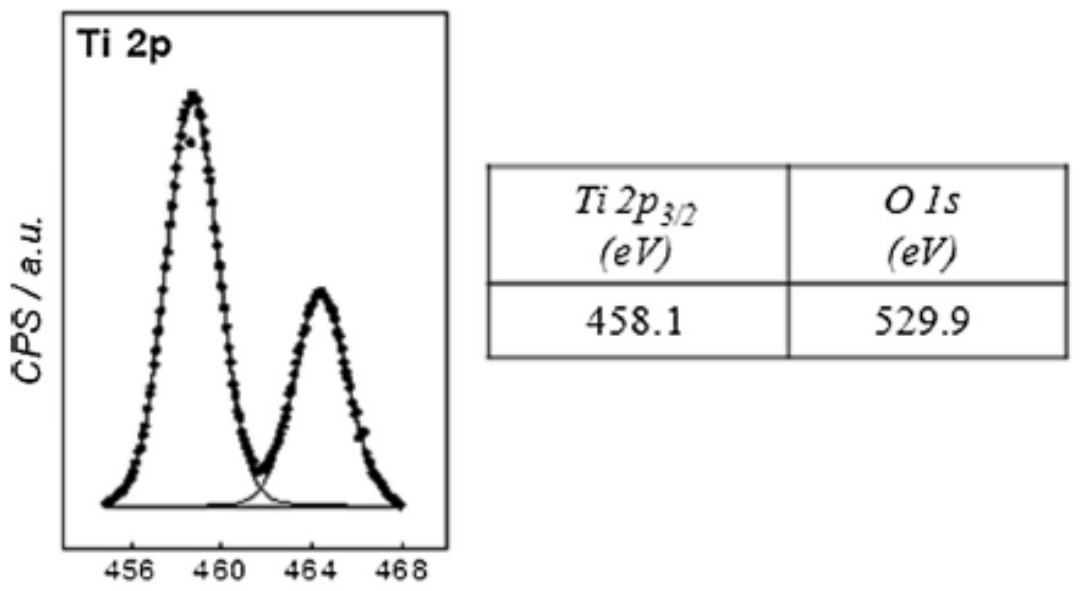

Fig. 4. Ti2p XPS spectrum of S6oo-R23. Table with the binding energies obtained from the XPS spectra for the sample S6oo-R23.

\subsection{DPPH antioxidant activity}

The decrease in DPPH content mediated by the different $\mathrm{TiO}_{2}$ samples is depicted in Fig. 5 . It can be seen that all powders show scavenging activity toward the DPPH radical. Moreover, independent of the calcination temperature of the powder, no significant differences were observed between the $\%$ of eliminated DPPH.

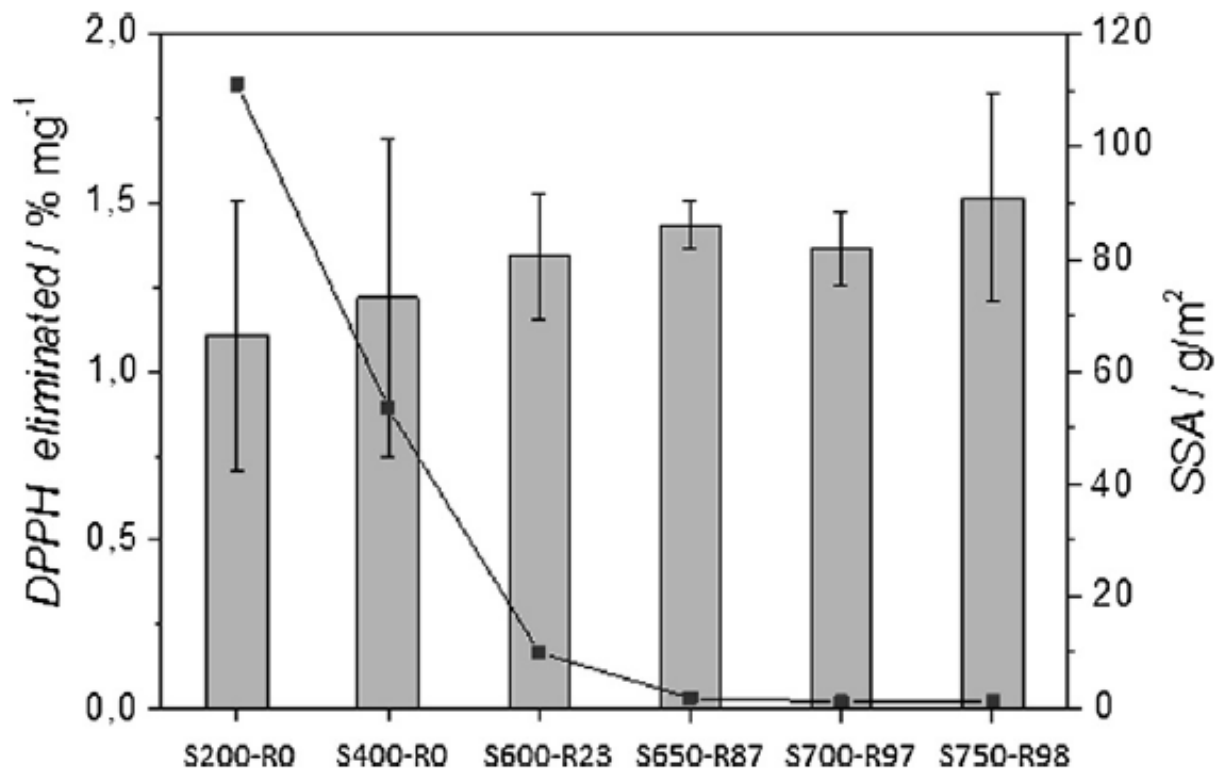

Version: Postprint (identical content as published paper) This is a self-archived document from i3S - Instituto de Investigação e Inovação em Saúde in the University of Porto Open Repository For Open Access to more of our publications, please visit http://repositorio-aberto.up.pt/ 
Fig. 5. Percentage of DPPH. eliminated by the $\mathrm{TiO}_{2}$ samples analyzed. Average $\pm \mathrm{SD}, \mathrm{n}=3$. SSA (specific surface area) of the samples.

Nevertheless, when one takes into consideration the scavenging capacity divided by the corresponding specific surface in each case (Fig. 6), to compare the percentage of radical species eliminated by $1 \mathrm{~m} 2$ of each phase composition, it can be concluded that the existence of a crystalline phase and the rutile-anatase ratio clearly determine the reactivity of the samples. Moreover, a critical percentage of rutile-anatase, above $23 \%$, is needed to improve the scavenging activity.

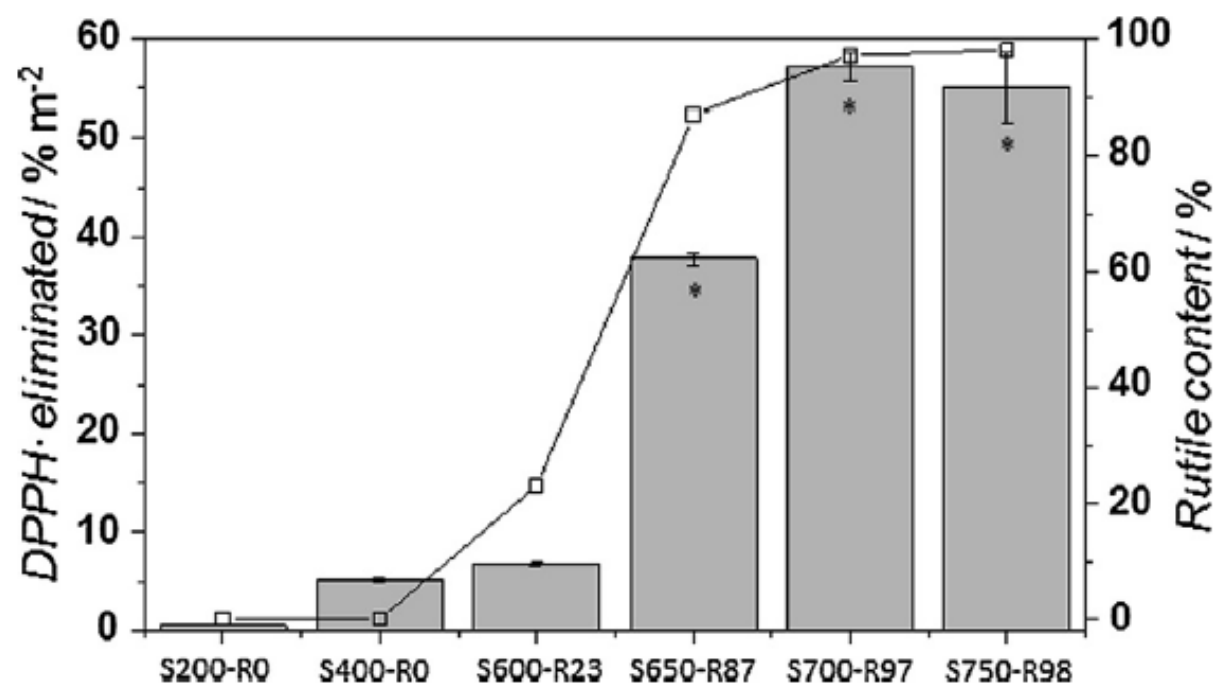

Fig. 6. Percentage of DPPH- eliminated, normalized by the SSAs of the prepared $\mathrm{TiO}_{2}$ powders (bars). Average $\pm S D, n=3 i$ * denotes significant differences $(p<0.05)$. Rutile content of the different samples (squared dots). 


\subsection{Peroxynitrite scavenging activity}

In a more biological approach, the ability of the $\mathrm{TiO}_{2}$ powders to deplete peroxynitrite was assessed by a SIN-1 based assay. Fig. 7 depicts the $\%$ of ONOO- depleted by each sample. It was observed that all the powders tested showed an ability to decrease the SIN-1 concentration after 30 min of reaction. Differently from what has been observed in the DPPH assay, the S600-R23 powders showed a significantly higher scavenging capacity.

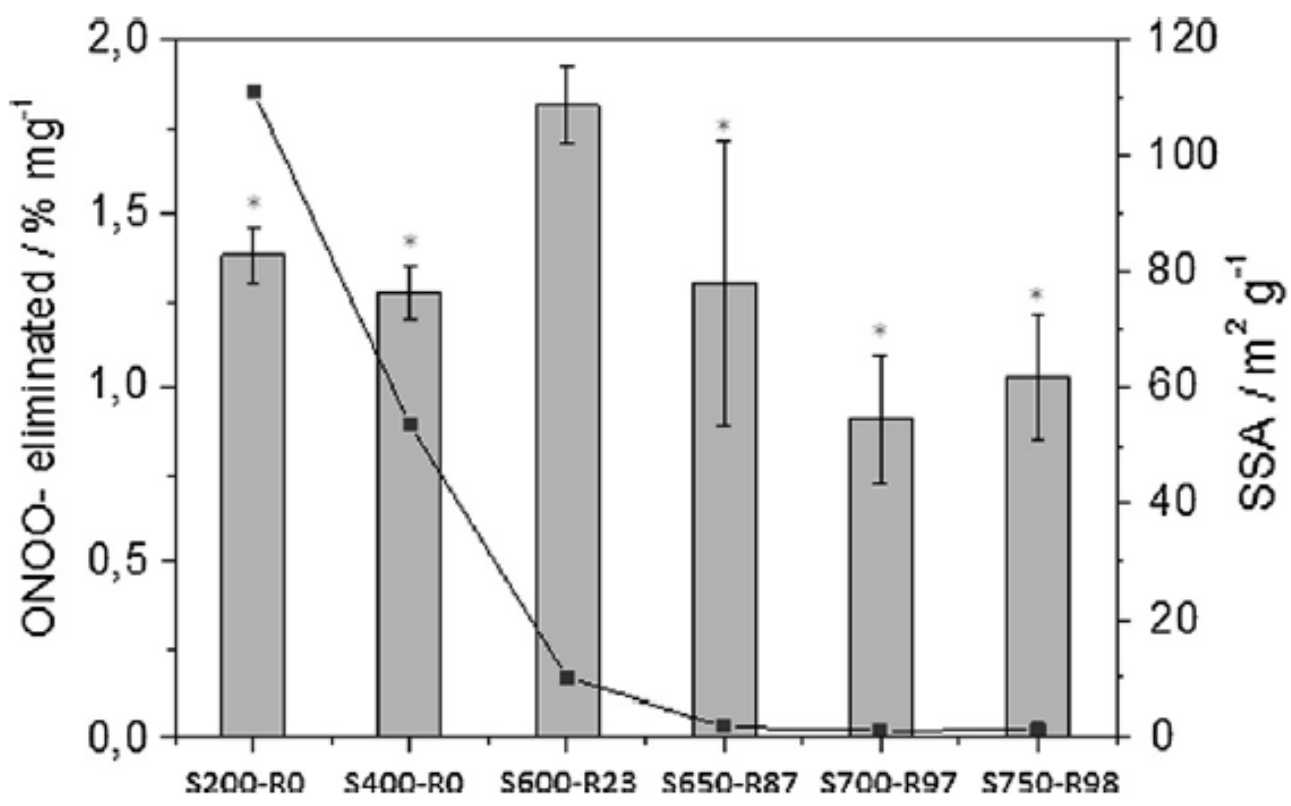

Fig. 7. Assessment of the peroxynitrite scavenging capacity of the prepared $\mathrm{TiO}_{2}$ powders. Average $\pm S D, n=3 i$ * denotes significant differences from S60o-R23 $(p<0.05)$.

However, when the percentage of peroxynitrite eliminated per specific surface $\left(\mathrm{m}_{2} \cdot g-1\right)$ was plotted (Fig. 8), the powders with the highest percentages of rutile phase are still the ones with the highest activity. It can also be concluded that the samples that have a rutile phase in their structure show higher activity per $\mathrm{m}_{2}$ that those in which $\mathrm{TiO}_{2}$ is in the anatase structure. 


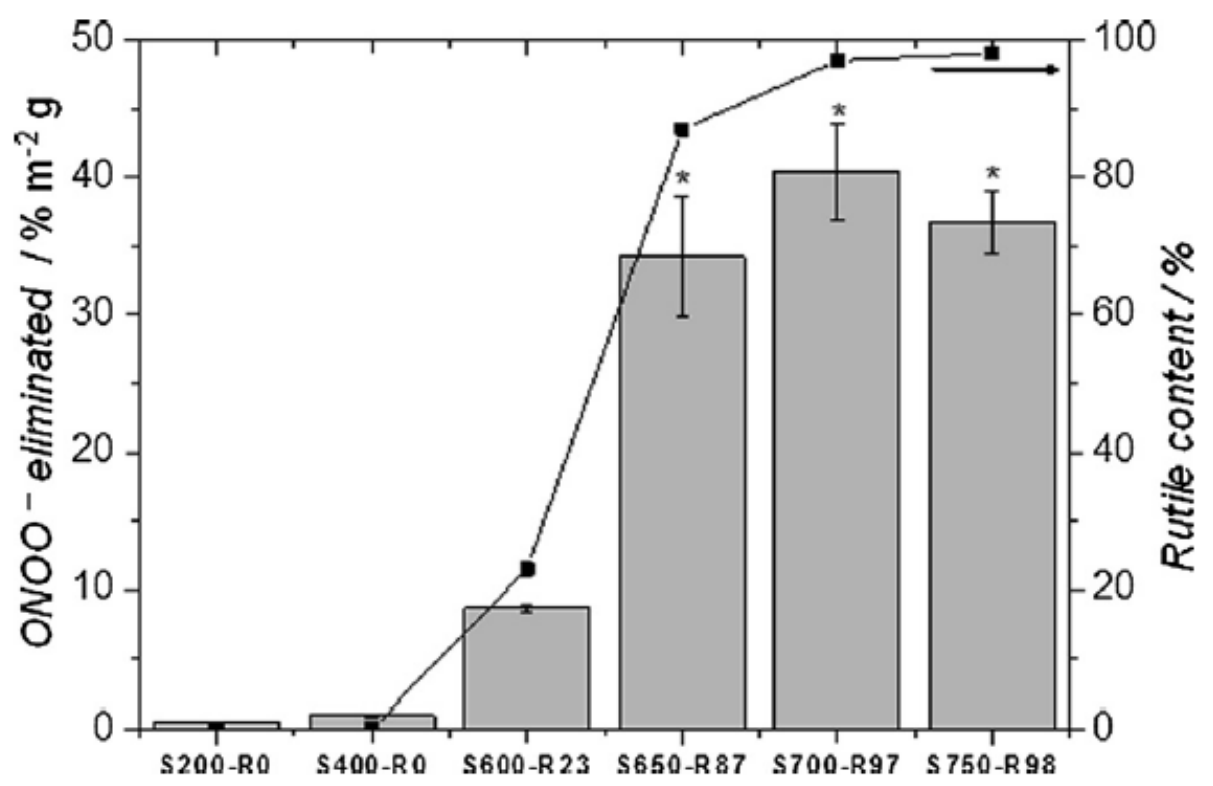

Fig. 8. Peroxynitrite eliminated per specific surface of the $\mathrm{TiO}_{2}$ powders. Average $\pm \mathrm{SD}, \mathrm{n}=3 i^{*}$ * denotes significant differences from the other samples $(p<0.05)$. Rutile content of the different samples (squared dots).

The scavenging activity of the $\mathrm{TiO}_{2}$ powders calcined at different temperatures has been successfully determined by an SIN-1 assay. The results achieved demonstrated that all the samples prepared were able to neutralize ONOO-. This molecule is known to be present in the body during the inflammatory response, and plays a key role in the further encapsulation of an implanted material [29]. Moreover, it was shown that the $\mathrm{TiO}_{2}$ powders exhibited this behavior under simulated physiological conditions. The measured reactivity was quite similar for all $\mathrm{TiO}_{2}$ powders, except in the case of $\mathrm{S} 600-\mathrm{R}_{23}$, which is slightly higher. Results divided by the SSA showed that the presence of the rutile phase at above $23 \%$ improves the scavenging activity of the material.

In addition, there is a correlation of the behavior of the powders with DPPH eliminated and with the results obtained in the SIN-1 assay. These findings confirm that while a higher \% of rutile is important to maintain the higher scavenging activity of the powders, the SSA also plays a key role in this activity; for this reason the most interesting powder is $\mathrm{S} 600-\mathrm{R} 23$, in which both factors are present and which therefore exhibits the highest scavenging activity in both assays.

Contreras evaluated the ROS/RNS scavenging activity of different titanate materials, proposing a set of reactions involved in the depletion of these species [12]. Their work was based on Tengvall's research, which assumed the existence of reduced $\mathrm{Ti}_{3}+$ states created during the integration of a $\mathrm{Ti}$ implant in the body [9]. These titanium reduced states play a key role in the reactions proposed in this work, as ONOO- is reduced to $\mathrm{NO}_{2}$ - through the oxidation of $\mathrm{Ti}_{3}+$ ions to $\mathrm{Ti}_{4}+$. In a later study, Canillas et al. explain that phases with a mixture of $\mathrm{Ti}_{4}+$ and $\mathrm{Ti}_{3}+$ present higher conductivities, which would improve the absorption of radical species onto the catalyst surface. This means that absorption or coordination of the reactive species could be an important parameter in scavenging activity [30]. 
While the existence of those species has been demonstrated in these works, in our research we have not used experimental methods to create Ti3 + states in our powders. Moreover, XPS results show a Tizp spectrum with one band associated only with the $\mathrm{Ti}_{4}+$ species, with no indication of any $\mathrm{Ti}_{3}+$. However, the powders are also able to neutralize ROS/RNS and other radicals. Thus, the differences found in the scavenging activity could be due to other parameters that affect the absorption or coordination of the reactive species to the catalytic surface.

From the results obtained, it is concluded that the initial reactions proposed in the literature for the depletion of ONOO- or DPPH can hardly take place in the prepared powders, for the simple reason that there is a lack of reduced titanium species in the material [11]. Nevertheless, the experimental assays carried out indicate that the reduction of ONOO- and DPPH is taking place in the samples. This prompted us to consider $\mathrm{TiO}_{2}$ not just as a redox species involved in the process, but as a catalyst that diminishes the reaction activation energy and favors those reactions. $\mathrm{TiO}_{2}$ is itself a catalytic material that acts in several known reactions [2], [29]. Here, we propose its catalytic contribution to the reduction reaction of those reactive compounds (peroxynitrite or DPPH) while other species are oxidized. Solvent molecules can be adsorbed onto the catalyst surface and this coordination or absorption onto the surface weakens the $\mathrm{OH}$ bond of the solvent (water or alcohol), aiding DPPH in catching the $\mathrm{H}$ to became acid peroxynitrite or $\mathrm{DPPH}_{2}$. In addition, the presence of anatase-rutile heterojunctions has been demonstrated through the HRTEM images in Fig. $2 \mathrm{~d}$ However, this has not been translated into an improvement in scavenging activity. It could support the idea that the mechanism would not involve electron exchange and transport in the catalysts as happens in photocatalysis.

It is known that $\mathrm{TiO}_{2}$ surfaces, both in anatase and rutile, have surface oxygen vacancies after simple calcination in air [31]. In fact, the thermodynamically more stable rutile shows a (1 10 ) facet. Along the [ $\left[\begin{array}{lll}0 & 0\end{array}\right]$ crystallographic direction, rows of sixfold-coordinated $\mathrm{Ti}$ atoms (Ti6c) alternate with fivefold-coordinated $\mathrm{Ti}$ atoms ( $\mathrm{Ti}_{5 \mathrm{C}}$ ). These defects generate two kinds of oxygen, threefoldcoordinated $\left(\mathrm{O}_{3} \mathrm{C}\right.$ ) in the main surface plane (as in the bulk) and bridging twofold-coordinated $\left(\mathrm{O}_{2} \mathrm{C}\right.$ or Ob) (Fig. 9) [32]. Similarly, after calcination in air, anatase surfaces are thermodynamically prone to show (101) facets. HRTEM studies have confirmed the existence of particles with those faces exposed in the anatase particles (Fig. 2). These facets show a characteristic terrace-like structure with surface ridges formed by ${ }_{5} \mathrm{Ti}_{5} \mathrm{C}$ and $\mathrm{O}_{2} \mathrm{C}$ (Fig. 9). These atoms are exposed to the interface and can interact more directly with water. Further, $\mathrm{O}_{3} \mathrm{C}$ atoms can be found on the surface interconnecting rows of Ti5c, and Ti6c is located at the bottom parts of the ridges. However, the latter two atom types are less important for interaction with water because their chemical valences are fully saturated by bonding within the $\mathrm{TiO}_{2}$ structure (Fig. 9b). [33] In both cases, anatase (llll) facets and rutile $\left(\begin{array}{lll}1 & 1 & 0\end{array}\right)$ facets, the $\mathrm{O}_{2} \mathrm{C}$ and $\mathrm{Ti}_{5 \mathrm{C}}$ atoms constitute natural reactive sites on the surface. For example, in the case of water media for SIN-1 assays, the first hydration layer is formed by water coordinated by oxygen atoms of water molecules (Ow) to surface Ti5c and water hydrogen-bonded by hydrogen atoms of water molecules $(\mathrm{Hw})$ to bridging $\mathrm{O}_{2} \mathrm{c}$ atoms. Similarly, with ethanol media (Et-OH) in DPPH assays, Et-OH can be coordinated by OEt to Ti5c or by HEt to $\mathrm{O}_{2} \mathrm{c}$. Moreover, the Ti5c sites that are found in both crystallographic phases can also be suitable sites for ONOO- and DPPH adsorption. 

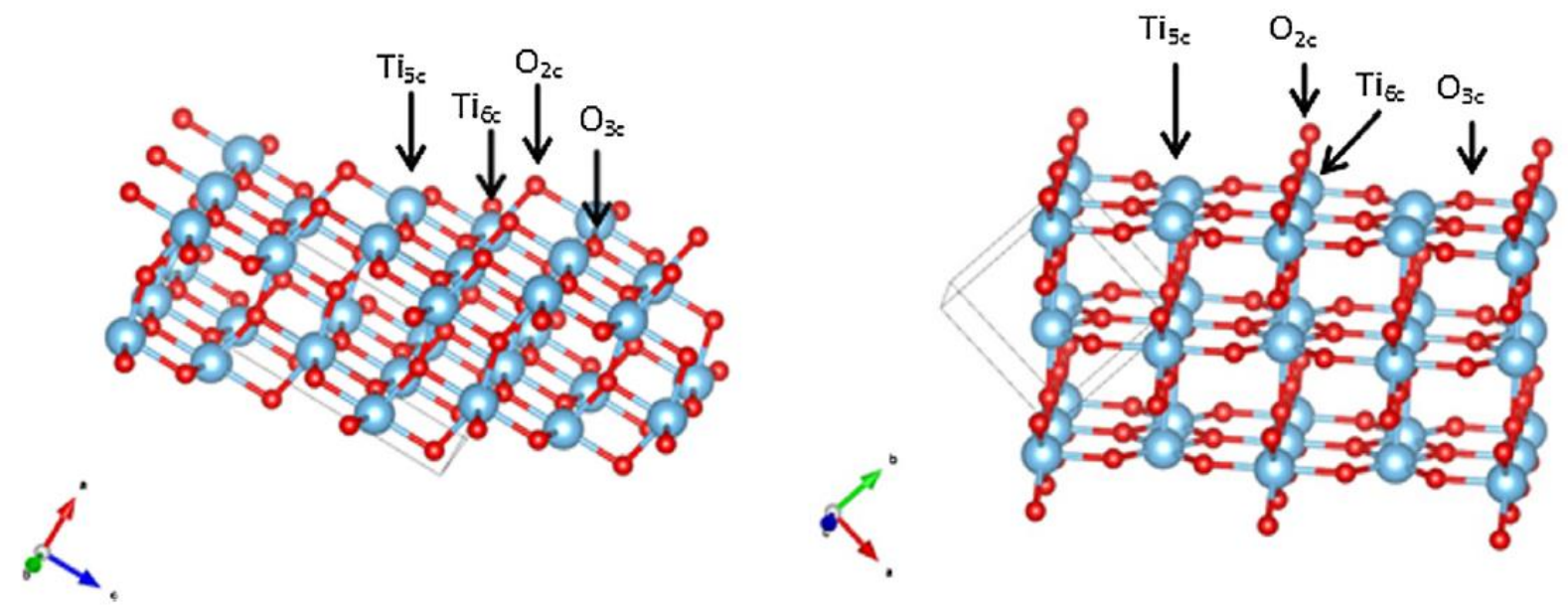

Fig. 9. Surface structure in anatase ( $\left.\begin{array}{lll}1 & 0 & 1\end{array}\right)$ and rutile $\left(\begin{array}{lll}1 & 1 & 0\end{array}\right)$ done with VESTA software.

It has been shown that the reaction can take place in the absence of anatase-rutile heterojunctions, through the results achieved for powders composed mainly of anatase or mainly of rutile (Fig. 5, Fig. 7), and in the absence of $\mathrm{Ti}_{3}+$ species on the surface. This is probably due to the concentration, in both cases, of $\mathrm{Ti}_{5 \mathrm{C}}$ and $\mathrm{O}_{2} \mathrm{C}$ active sites to carry out the adsorption and facilitate the neutralization reaction of the radicals [34]. When the percentage of radical species neutralized is divided by the corresponding specific surface areas of powders (Fig. 6, Fig. 8), it can be observed that the higher the percentage of rutile in the sample, the higher is the scavenger power. This can be explained in accord with the study by Futera et al. oft oscillating electric-field effects on adsorbed water at rutile and anatase $\mathrm{TiO}_{2}$ surfaces [35]. These authors demonstrate according to diffusivity measurements that water is strongly adsorbed onto (110) rutile facets, while weaker adsorption can be expected on ( $\left.\begin{array}{lll}1 & 0 & 1\end{array}\right)$ anatase surfaces. On ( 110 ) rutile facets, water is strongly bonded by Owater (OW) to Ti5c and the confinement is intensified by hydrogen bonding to neighboring $\mathrm{O}_{2} \mathrm{C}$ atoms. However, on $\left(\begin{array}{lll}1 & 0 & 1\end{array}\right)$ anatase facets, Ti5c surface atoms are located on the same row as the exposed $\mathrm{O}_{2} \mathrm{C}$ atoms and therefore they are less accessible to water. Similar adsorption could be expected for $\mathrm{Et}-\mathrm{OH}$ media on the $\mathrm{TiO}_{2}$ surfaces, leaving more accessible to the hydrogen of $\mathrm{OH}$ groups of $\mathrm{H}_{2} \mathrm{O}$ or $\mathrm{Et}-\mathrm{OH}$. Then they will be more easily captured by SIN-1 and DPPH, leading to their neutralization. The increase of scavenging activity with the percentage of rutile would be associated with the stronger adsorption of $\mathrm{H}_{2} \mathrm{O}$ or EtOH molecules onto the surface of $\mathrm{TiO}_{2}$.

\section{Conclusions}

In this work, the free radical scavenging activity of different $\mathrm{TiO}_{2}$ powders has been tested and compared. The influence of physicochemical features of the $\mathrm{TiO}_{2}$ powders has been considered as a possible explanation for the different behavior found. The results establish $\mathrm{TiO}_{2}$ as a good radical scavenger, with scavenging activity depending on the ceramic processing method used for its preparation. It has been demonstrated that the rutile content is responsible for higher anti-ROS/RNS activity and that the specific surface area plays a key role in the reactivity of the powders. For the first time, this work has shown that $\mathrm{TiO}_{2}$ powders with no electronic defects ( $\mathrm{Ti}_{3}+$ species) can neutralize ROS/RNS. Moreover, heterojunctions do not promote an increase in scavenging activity. For these 
reasons, we suggest a catalytic process where $\mathrm{Ti}_{5} \mathrm{C}$ and $\mathrm{O}_{2} \mathrm{C}$ act as active sites in the catalytic mechanism. Furthermore, the disposition of those active sites in the surfaces of preferential facets of anatase and rutile could explain the higher reactivity of rutile due to stronger adsorption of $\mathrm{H}_{2} \mathrm{O}$ or $\mathrm{EtOH}$ molecules onto its surface than onto that of anatase. This coordination would made the catching of the hydrogen of $\mathrm{H}_{2} \mathrm{O}$ or EtOH to react with SIN-1 or DPPH molecules and reduce them easier.

\section{Acknowledgements}

The authors thank Dr. Del Campo for his help with band gap measurements and Dr. GonzalezCalatayud for his helpful discussions. They acknowledge the European Project NERBIOS (NEST/STREP, FP6, 028473-2), the Conselho de Reitores das Universidades Portuguesas (Acções Integradas Luso-Espanholas, E-21/11), the Fundación General del CSIC, and La Obra Social LaCaixa for the financial support received (Proyectos Envejecimiento Cero, ref. 2001). M. Canillas acknowledges the JAE-CSIC Ph.D. fellowship received and B. Moreno acknowledges the Fondo Social Europeo and the JAEDoc program for their financial support.

\section{REFERENCES}

[1] R. Kohen, A. Nyska, Oxidation of biological systems: oxidative stress phenomena, antioxidants, redox reactions, and methods for their quantification, Toxicol. Pathol. 30 (6) (2002) 620 .

[2] T.J. Guzik, R. Korbut, T. Adamek-Guzik, Nitric oxide and superoxide in inflammation and immune regulation, J. Phys. Pharm. 54 (4) (2003) 469.

[3] B.F. Oliveira, J.A. Nogueira-Machado, M.M. Chaves, The role of oxidative stress in the aging process, Sci. World J. 10 (2010) 1121.

[4] H.Y. Chung, H.J. Kim, J.W. Kim, B.P. Yu, The inflammation hypothesis of aging, Ann. N.Y. Acad. Sci. 928 (2001) 327.

[5] E.G. McGeer, P.L. McGeer, Brain inflammation in Alzheimer disease and the therapeutic implications, Curr. Pharm. Des. 5 (1999) 821.

[6] T.J. Guzik, N. West, R. Pillai, D.P. Taggart, K.M. Channon, Nitric oxide modulates superoxide release and peroxynitrite formation in human blood vessels, Hypertension 39 (2002) 1088.

[7] W. Dröge, Free radicals in the physiological control of cell function, Physiol. Rev. 82 (1) (2002) 47.

[8] R. Suzuki, J.A. Frangos, Inhibition of inflammatory species by titanium surfaces, Clin. Orthopaed. Relat. Res. 362 (1999) 280 .

[9] P. Tengvall, I. Lundström, L. Sjöqvist, H. Elwing, Interaction between hydrogen peroxide and titanium: a possible role in the biocompatibility of titanium, Biomaterials 10 (2) (1989) 118 .

[10] R. Suzuki, J. Muyco, J. McKittrick, J.A. Frangos, Reactive oxygen species inhibited by titanium oxide coatings, J. Biomed. Mater. Res. Part A 66 (2) (2003) 396.

[11] H. Sahlin, R. Contreras, D.F. Gaskill, L.M. Bjursten, J.A. Frangos, Antiinflammatory properties of micropatterned titanium Coatings, J. Biomed. Mater. Res. Part A 77 (1) (2005) 43.

[12] R. Contreras, H. Sahlin, J.A. Frangos, Titanate biomaterials with enhanced antiinflammatory properties, J. Biomed. Mater. Res. Part A 80 (2) (2006) 480.

\section{.}


[13] J.E. Graves, D. Pletcher, R.L. Clarke, F.C. Walsh, The electrochemistry of Magnéli phase titanium oxide ceramic electrodes Part I. The deposition and properties of metal coatings, J. Appl. Electrochem. 21 (1991) 848.

[14] H. Zhang, M. Finnegan, J.F. Banfield, Preparing single-phase nanocrystalline anatase from amorphous titania with particle sizes tailored by temperature, Nano Lett. 1 (2001) 81.

[15] H. Zhang, J.F. Banfield, Understanding polymorphic phase transformation behavior during growth of nanocrystalline aggregates: insights from TiO2, J. Phys. Chem. B 104 (2000) 3491.

[16] A.S. Barnard, L.A. Curtis, Prediction of $\mathrm{TiO}_{2}$ nanoparticle phase and shape transitions controlled by surface chemistry, Nanoletters 5 (7) (2005) 1261.

[17] T.A. Egerton, J.A. Mattinson, The influence of platinum on UV and 'visible' photocatalysis by rutile and Degussa P25, J. Photochem. PhotoBiol. A 186 (2007) 115

[18] M. Carballo-Vila, B. Moreno-Burriel, E. Chinarro, J.R. Jurado, N. Casañ-Pastor, J. E. Collazos-Castro, Titanium oxide as substrate for neural cell growth, J. Biomed. Mater. Res. A 1 (2008) 94-105.

[19] M. Carballo-Vila, B. Moreno-Burriel, E. Chinarro, J.R. Jurado, N. Casañ-Pastor, J. E. Collazos-Castro, Titanium oxide as substrate for neural cell growth, J. Biomed. Mater. Res. A 1 (2008) 94-105.

[20] J. Tauc, Optical properties and electronic structure of amorphous Ge and Si, J. Mater. Res. Bull. 3 (1968) 37.

[21] R.A. Van Leeuwen, C.-J. Hung, D.R. Kammler, J.A.J. Switzer, Optical and electronic transport properties of electrodeposited thallium(III) oxide films, Phys. Chem. 99 (41) (1995) 15247.

[22] C.P. LeBel, H. Ischiropoulos, S.C. Bondy, Evaluation of the probe $2^{\prime}, 7^{\prime}$ - dichlorofluorescin as an indicator of reactive oxygen species formation and oxidative stress, Chem. Res. Toxicol. 5 (1992) 227.

[23] B. Ozcelik, J.H. Lee, D.B. Min, Effects of light, oxygen, and pH on the absorbance of 2,2-Diphenyl-1picrylhydrazyl, Food Chem. Toxicol. 68 (2003) 487.

[24] M. Leggas, Y. Zhuang, J. Welden, Z. Self, C.M. Waters, C.F. Stewart, Microbore HPLC method with online microdialysis for measurement of topotecan lactone and carboxylate in murine CSF, J. Pharm. Sci. 93 (9) (2004) 2284 .

[25] X.-H. Zhang, X. Zheng, Y. Cheng, G.-H. Li, X.-P. Chen, J.-H. Zheng, Formation of rutile fasciculate zone induced by sunlight irradiation at room temperature and its hemocompatibility, Mater. Sci. Eng. C 33 (2013) 3289.

[26] P.-O. Wang, Y. Bai, J.-Y. Liu, Z. Fan, Y.-O. Hu, One-pot synthesis of rutile $\mathrm{TiO}_{2}$ nanoparticle modified anatase $\mathrm{TiO}_{2}$ nanorods toward enhanced photocatalytic reduction of $\mathrm{CO}_{2}$ into hydrocarbon fuels, Catal. Comm. (2012) 29-185.

[27] R. Menzel, A. Duerrbeck, E. Liberti, YauH. Chun, D. McComb, Hydrothermal Stability of \{001\} Faceted Anatase TiO2, Shaffer M.S.P, Chem. Mater. 25 (2013) 2137.

[28] W.-N. Zhao, S.-C. Zhu, Y.-F. Li, Z.-P. Liu, Three-phase junction for modulating electron-hole migration in anatase-rutile photocatalysts, Chem. Sci. 6 (2015) 3483.

[29] R.D. Jayant, M.J. McShane, R. Srivastava, In vitro and in vivo evaluation of antiinflammatory agents using nanoengineered alginate carriers: towards localized implant inflammation suppression, Int. Journ. Pharm. 403 (12) (2011) 268 .

[30] M. Canillas, E. Chinarro, A. Pego, B. Moreno, Scavenging activity of Magnéli phases as a function of Ti4+/Ti3+ ratios, Chem. Commun. (2017).

[31] Y. Yan, G. Wu, N. Guan, L. Li, Z. Li, X. Cao, Understanding the effect of surface/ bulk defects on the photocatalytic activity of TiO2: anatase versus rutile, Phys. Chem. Chem. Phys. 15 (2013) 10978.

[32] U. Diebold, Structure and properties of TiO2 surfaces: a brief review, Appl. Phys. A 76 (2003) 681. 
[33] J. Pan, G. Liu, G. Qing, M. Lu, H.-M. Cheng, Angew, On the true photoreactivity order of 001$\}$, $\{010\}$, and $\{101$ facets of anatase $\mathrm{TiO}_{2}$ crystals, Chem. Int. Ed. 50 (2011) 2133.

[34] Z. Futera, N.J. English, Exploring rutile (110) and anatase (101) TiO2 water interfaces by reactive force-field simulations, Phys. Chem. C 121 (2017) 6701-6711.

[35] Z. Futera, N.J. English, Oscillating electric-field effects on adsorbed-water at rutile- and anatase-TiO2 surfaces, J. Chem. Phys. 145 (2016). 